\title{
Some Notes on the Existence of Solution for Ordinary Differential Equations via Fixed Point Theory
}

\author{
Fei He \\ School of Mathematical Sciences, Inner Mongolia University, Hohhot 010021, China \\ Correspondence should be addressed to Fei He; hefei@imu.edu.cn
}

Received 27 June 2014; Accepted 25 July 2014; Published 14 October 2014

Academic Editor: Wei-Shih Du

Copyright (C) 2014 Fei He. This is an open access article distributed under the Creative Commons Attribution License, which permits unrestricted use, distribution, and reproduction in any medium, provided the original work is properly cited.

We establish a fixed point theorem with $w$-distance for nonlinear contractive mappings in complete metric spaces. As applications of our results, we derive the existence and uniqueness of solution for a first-order ordinary differential equation with periodic boundary conditions. Here, we need not assume that the equation has a lower solution.

\section{Introduction and Preliminaries}

The main purpose of this paper is to obtain the existence and uniqueness of solution for a periodic boundary value problem. This topic has been considered in [1-11] and the references therein. A powerful tool to solve the problem is the fixed point theorem in partially ordered metric spaces. On the existing research results, admitting the existence of a lower solution is necessary. In this paper, we establish a fixed point theorem for $w$-distance contraction type maps in complete metric spaces without partially ordered structure. From this, we obtain some results on the existence and uniqueness for ordinary differential equations. We will see that the assumption of the existence of a lower solution can be removed. In particular, we also show that under assumptions of a recent result in [10] the equation has a unique zero solution.

In this note, we consider the following periodic boundary value problem:

$$
\begin{gathered}
u^{\prime}(t)=f(t, u(t)), \quad \text { if } t \in I=[0, T] ; \\
u(0)=u(T),
\end{gathered}
$$

where $T>0$ and $f: I \times \mathbf{R}$ is a continuous function.

Definition 1. A lower solution for (1) is a function $\alpha \in C^{1}(I)$ such that

$$
\begin{gathered}
\alpha^{\prime}(t) \leq f(t, \alpha(t)) \quad \text { for } t \in I, \\
\alpha(0) \leq \alpha(T) .
\end{gathered}
$$

Let $X=C(I)$ be the set of all real continuous functions on a closed interval $I$. We endow $X$ with the norm

$$
\|u\|=\max _{t \in I}|u(t)|,
$$

for all $u \in X$. Obviously, this space is a Banach space and the norm induces a complete metric on $X$ as follows:

$$
d(u, v)=\|u-v\|=\max _{t \in I}|u(t)-v(t)|
$$

for all $u, v \in X$.

Let $\mathscr{B}$ denote the class of those functions $\beta:[0, \infty) \rightarrow$ $[0,1)$ which satisfy the condition

$$
\beta\left(t_{n}\right) \longrightarrow 1 \text { implies } t_{n} \longrightarrow 0 \text {. }
$$

Let $\mathscr{A}$ denote the class of the functions $\phi:[0, \infty) \rightarrow[0, \infty)$ which have the following properties:

(i) $\phi$ is increasing;

(ii) for each $x>0, \phi(x)<x$;

(iii) $\beta(x)=\phi(x) / x \in \mathscr{B}$.

For example, $\phi(t)=\mu t$, where $0 \leq \mu<1, \phi(t)=t /(1+t)$, and $\phi(t)=\ln (1+t)$ are in $\mathscr{A}$.

Recently, Amini-Harandi and Emami [4] proved the following existence theorem. 
Theorem 2 (see [4, Theorem 3.1]). Consider problem (1) with $f: I \times \mathbf{R} \rightarrow \mathbf{R}$ continuous and suppose that there exists $\lambda>0$ such that for $x, y \in \mathbf{R}$ with $y \geq x$,

$$
0 \leq f(t, y)+\lambda y-[f(t, x)+\lambda x] \leq \lambda \phi(y-x),
$$

where $\phi \in \mathscr{A}$. Then the existence of a lower solution of (1) provides the existence of a unique solution of (1).

Then, Caballero et al. [6] also give an existence theorem.

Theorem 3 (see [6, Theorem 3.2]). Consider problem (1) with $f: I \times \mathbf{R} \rightarrow \mathbf{R}$ continuous and suppose that there exist $\lambda, \alpha>0$ with

$$
\alpha \leq\left(\frac{2 \lambda\left(e^{\lambda T}-1\right)}{T\left(e^{\lambda T}+1\right)}\right)^{1 / 2}
$$

such that for $x, y \in \mathbf{R}$ with $y \geq x$

$$
0 \leq f(t, y)+\lambda y-[f(t, x)+\lambda x] \leq \alpha \sqrt{(y-x) \phi(y-x)},
$$

where $\phi \in \mathscr{A}$. Then the existence of a lower solution of (1) provides the existence of a solution of (1). result.

Very recently, Hussain et al. [10] obtain the following

Theorem 4 (see [10, Section 3]). Consider problem (1) with $f: I \times \mathbf{R} \rightarrow \mathbf{R}$ continuous and suppose that there exists $\lambda>0$ such that for $x, y \in C(I)$

$$
\begin{aligned}
& |f(t, x(t))+\lambda x(t)|+|f(t, y(t))+\lambda y(t)| \\
& \quad \leq \frac{\lambda}{2^{p-1}} p \sqrt[p]{\ln \left(\frac{a}{2^{p-1}}(|x(t)|+|y(t)|)^{p}+1\right)},
\end{aligned}
$$

where $0 \leq a<1$ and $p>1$. Then the existence of a lower solution of (1) provides the existence of a solution of (1).

Remark 5. If we take $x(t)=y(t)=\theta(t)=0$ for all $t \in I$ in the condition (9), then we deduce that

$$
f(t, \theta(t))=0 \quad \forall t \in I .
$$

This means that $\theta(t)$ is a solution of (1) (of course, a lower solution of (1)). In Section 3, we will show this fact again; see Remark 17.

Now, let us recall the concept of $w$-distance, which was introduced by Kada et al. [12].

Definition 6. Let $X$ be a metric space with metric $d$. Then a function $p: X \times X \rightarrow[0, \infty)$ is called a $w$-distance on $X$ if the following are satisfied:

(w1) $p(x, z) \leq p(x, y)+p(y, z)$, for any $x, y, z \in X$;

(w2) for any $x \in X, p(x, \cdot): X \rightarrow[0, \infty)$ is lower semicontinuous; that is, $p\left(x, y_{0}\right) \leq \liminf _{n \rightarrow \infty} p\left(x, y_{n}\right)$, whenever $y_{n} \in X$ and $y_{n} \rightarrow y_{0}$; (w3) for any $\varepsilon>0$, there exists $\delta>0$ such that $p(z, x) \leq \delta$ and $p(z, y) \leq \delta$ imply $d(x, y) \leq \varepsilon$.

Let us give some basic examples of $w$-distances (see [12]).

Example 7. Let $(X, d)$ be a metric space. Then the metric $d$ is a $w$-distance on $X$.

Example 8. Let $X$ be a normed space with norm $\|\cdot\|$. Then the function $p: X \times X \rightarrow[0, \infty)$ defined by

$$
p(x, y)=\|x\|+\|y\|
$$

for every $x, y \in X$ is a $w$-distance on $X$.

Example 9. Let $X$ be a normed space with norm $\|\cdot\|$. Then the function $p: X \times X \rightarrow[0, \infty)$ defined by

$$
p(x, y)=\|y\|
$$

for every $x, y \in X$ is a $w$-distance on $X$.

We also need the following example in Section 3.

Example 10. Let $X=C(I)$ be endowed with the norm

$$
\|u\|=\max _{t \in I}|u(t)|,
$$

for all $u \in X$. We easily deduce that the function $p: X \times X \rightarrow$ $[0, \infty)$ defined by

$$
p(x, y)=\max _{t \in I}(|x(t)|+|y(t)|)
$$

for every $x, y \in X$ is a $w$-distance on $X$.

The following lemma has been proved in [12].

Lemma 11. Let $X$ be a metric space with metric $d$ and let $p$ be a $w$-distance on $X$. Let $\left\{x_{n}\right\}$ and $\left\{y_{n}\right\}$ be sequences in $X$, let $\left\{\alpha_{n}\right\}$ and $\left\{\beta_{n}\right\}$ be sequences in $[0, \infty)$ converging to 0 , and let $x, y, z \in X$. Then the following hold.

(i) If $p\left(x_{n}, y\right) \leq \alpha_{n}$ and $p\left(x_{n}, z\right) \leq \beta_{n}$ for any $n \in \mathbf{N}$, then $y=z$. In particular, if $p(x, y)=0$ and $p(x, z)=0$, then $y=z$.

(ii) If $p\left(x_{n}, y_{n}\right) \leq \alpha_{n}$ and $p\left(x_{n}, z\right) \leq \beta_{n}$ for any $n \in \mathbf{N}$, then $\left\{y_{n}\right\}$ converges to $z$.

(iii) If $p\left(x_{n}, x_{m}\right) \leq \alpha_{n}$ for any $n, m \in \mathbf{N}$ with $m>n$, then $\left\{x_{n}\right\}$ is a Cauchy sequence.

\section{Fixed Point Results with $w$-Distance}

We are now ready to state and prove our main theorem.

Theorem 12. Let $(X, d)$ be a complete metric space and let $p$ be a $w$-distance on $X$. Let $f: X \rightarrow X$ be a map and suppose that there exists $\beta \in \mathscr{B}$ such that

$$
p(f x, f y) \leq \beta(p(x, y)) p(x, y),
$$

for all $x, y \in X$. Then $f$ has a unique fixed point $z$ in $X$ and $p(z, z)=0$. 
Proof. Starting with an arbitrary $x_{0} \in X$, we construct by induction a sequence $\left\{f^{n}\left(x_{0}\right)\right\}$ in $X$. Put $x_{n}=f^{n}\left(x_{0}\right), n=$ $1,2, \ldots$.. have

Applying the condition (15) with $x=x_{n}$ and $y=x_{n+1}$, we

$$
\begin{aligned}
p\left(x_{n+1}, x_{n+2}\right) & =p\left(f x_{n}, f x_{n+1}\right) \\
& \leq \beta\left(p\left(x_{n}, x_{n+1}\right)\right) p\left(x_{n}, x_{n+1}\right) \\
& \leq p\left(x_{n}, x_{n+1}\right) .
\end{aligned}
$$

Therefore $\left\{p\left(x_{n}, x_{n+1}\right)\right\}$ is a decreasing sequence and bounded below and hence $\lim _{n \rightarrow \infty} p\left(x_{n}, x_{n+1}\right)=r \geq 0$.

Let us prove that $r=0$. Assume that $r>0$. From (15), we obtain

$$
\frac{p\left(x_{n+1}, x_{n+2}\right)}{p\left(x_{n}, x_{n+1}\right)} \leq \beta\left(p\left(x_{n}, x_{n+1}\right)\right)<1, \quad n=1,2, \ldots
$$

Taking limit when $n \rightarrow \infty$, the above inequality yields $\lim _{n \rightarrow \infty} \beta\left(p\left(x_{n}, x_{n+1}\right)\right)=1$, and since $\beta \in \mathscr{B}$ this implies $r=0$. Thus $\lim _{n \rightarrow \infty} p\left(x_{n}, x_{n+1}\right)=0$. Analogously, it can be proved that $\lim _{n \rightarrow \infty} p\left(x_{n+1}, x_{n}\right)=0$.

Now, we show that

$$
p\left(x_{n}, x_{m}\right) \longrightarrow 0 \text { as } m>n \longrightarrow \infty \text {. }
$$

Suppose that this is not true. Then there exists $\varepsilon_{0}>0$ for which we can find subsequences $\left\{x_{n_{k}}\right\}$ and $\left\{x_{m_{k}}\right\}$ of $\left\{x_{n}\right\}$ with $m_{k}>n_{k}>k$ such that

$$
p\left(x_{n_{k}}, x_{m_{k}}\right) \geq \varepsilon_{0}, \quad k=1,2, \ldots
$$

By the triangle inequality and the condition (15), for every $k \in \mathbf{N}$,

$$
\begin{aligned}
p\left(x_{n_{k}}, x_{m_{k}}\right) \leq & p\left(x_{n_{k}}, x_{n_{k}+1}\right)+p\left(x_{n_{k}+1}, x_{m_{k}+1}\right) \\
& +p\left(x_{m_{k}+1}, x_{m_{k}}\right) \\
\leq & p\left(x_{n_{k}}, x_{n_{k}+1}\right)+\beta\left(p\left(x_{n_{k}}, x_{m_{k}}\right)\right) p\left(x_{n_{k}}, x_{m_{k}}\right) \\
& +p\left(x_{m_{k}+1}, x_{m_{k}}\right) .
\end{aligned}
$$

From this, we obtain

$$
\begin{aligned}
1-\beta & \left(p\left(x_{n_{k}}, x_{m_{k}}\right)\right) \\
& \leq p\left(x_{n_{k}}, x_{m_{k}}\right)^{-1} \cdot\left[p\left(x_{n_{k}}, x_{n_{k}+1}\right)+p\left(x_{m_{k}+1}, x_{m_{k}}\right)\right] \\
& \leq \varepsilon_{0}^{-1} \cdot\left[p\left(x_{n_{k}}, x_{n_{k}+1}\right)+p\left(x_{m_{k}+1}, x_{m_{k}}\right)\right] .
\end{aligned}
$$

Letting $k \rightarrow \infty$ in the above inequality, using $\lim _{n \rightarrow \infty} p\left(x_{n}\right.$, $\left.x_{n+1}\right)=0$ and $\lim _{n \rightarrow \infty} p\left(x_{n+1}, x_{n}\right)=0$, we deduce that

$$
\lim _{k \rightarrow \infty} \beta\left(p\left(x_{n_{k}}, x_{m_{k}}\right)\right)=1 \text {. }
$$

But since $\beta \in \mathscr{B}$ we get $\lim _{k \rightarrow \infty} p\left(x_{n_{k}}, x_{m_{k}}\right)=0$. This is a contradiction and hence we have shown that (18) holds.

From (18) and Lemma 11(iii), it follows that $\left\{x_{n}\right\}$ is a Cauchy sequence in $X$. Since $(X, d)$ is a complete metric space, there exists a $z \in X$ such that $\lim _{n \rightarrow \infty} x_{n}=z$.

Next, we prove that $z$ is a fixed point of $f$. To the end, we prove that $\lim _{n \rightarrow \infty} p\left(x_{n}, z\right)=0$ and $\lim _{n \rightarrow \infty} p\left(x_{n}, f z\right)=0$. Let $\varepsilon>0$ be given. Using (18), there exists $n_{0} \in \mathbf{N}$ such that $p\left(x_{n}, x_{m}\right)<\varepsilon$ for all $m>n \geq n_{0}$. Letting $m \rightarrow \infty$, by (w2) we have $p\left(x_{n}, z\right) \leq \liminf _{m \rightarrow \infty} p\left(x_{n}, x_{m}\right) \leq \varepsilon$ for all $n \geq n_{0}$. This means that $\lim _{n \rightarrow \infty} p\left(x_{n}, z\right)=0$. Applying the condition (15) with $x=x_{n}$ and $y=z$, we have

$$
\begin{aligned}
p\left(x_{n+1}, f z\right) & =p\left(f x_{n}, f z\right) \leq \beta\left(p\left(x_{n}, z\right)\right) p\left(x_{n}, z\right) \\
& \leq p\left(x_{n}, z\right) .
\end{aligned}
$$

Due to $\lim _{n \rightarrow \infty} p\left(x_{n}, z\right)=0$, it follows that $\lim _{n \rightarrow \infty} p\left(x_{n}\right.$, $f z)=0$. According to Lemma 11(i), we obtain $f z=z$; that is, $z$ is a fixed point of $f$.

To prove the uniqueness of the fixed point of $f$, let us suppose that $y$ is another fixed point of $f$. Using the condition (15), we get

$$
\begin{aligned}
p\left(x_{n+1}, y\right) & =p\left(f x_{n}, f y\right) \leq \beta\left(p\left(x_{n}, y\right)\right) p\left(x_{n}, y\right) \\
& \leq p\left(x_{n}, y\right) .
\end{aligned}
$$

Therefore $\left\{p\left(x_{n}, y\right)\right\}$ is a decreasing sequence and bounded below and hence $\lim _{n \rightarrow \infty} p\left(x_{n}, y\right)=s \geq 0$. Suppose that $s>$ 0 . Using the condition (15), we have

$$
\frac{p\left(x_{n+1}, y\right)}{p\left(x_{n}, y\right)} \leq \beta\left(p\left(x_{n}, y\right)\right)<1, \quad n=1,2, \ldots .
$$

Taking limit when $n \rightarrow \infty$, the above inequality yields $\lim _{n \rightarrow \infty} \beta\left(p\left(x_{n}, y\right)\right)=1$, and since $\beta \in \mathscr{B}$ this implies $s=0$. Then $\lim _{n \rightarrow \infty} p\left(x_{n}, y\right)=0$. Combining this and $\lim _{n \rightarrow \infty} p\left(x_{n}, z\right)=0$, we get by Lemma 11(i) that $y=z$.

Finally, we prove $p(z, z)=0$ when $z$ is a fixed point of $f$. Applying the condition (15) with $x=z$ and $y=z$, we obtain

$$
p(z, z)=p(f z, f z) \leq \beta(p(z, z)) p(z, z)
$$

and hence $(1-\beta(p(z, z))) \cdot p(z, z)=0$. Now that $\beta \in \mathscr{B}$, it follows that $p(z, z)=0$.

The following corollary is immediate result from Theorem 12 and Example 7.

Corollary 13. Let $(X, d)$ be a complete metric space. Let $f$ : $X \rightarrow X$ be a map and suppose that there exists $\beta \in \mathscr{B}$ such that

$$
d(f x, f y) \leq \beta(d(x, y)) d(x, y)
$$

for all $x, y \in X$. Then $f$ has a unique fixed point $z$ in $X$.

\section{Application to Ordinary Differential Equations}

Now, we prove some results on the existence of solution for problem (1). 
Theorem 14. Consider problem (1) with $f$ continuous and suppose that there exists $\lambda>0$ such that for any $x, y \in \mathbf{R}$

$$
|f(t, y)+\lambda y-[f(t, x)+\lambda x]| \leq \lambda \phi(|y-x|),
$$

where $\phi \in \mathscr{A}$. Then there exists a unique solution for (1).

Proof. Problem (1) can be rewritten as

$$
\begin{gathered}
u^{\prime}(t)+\lambda u(t)=f(t, u(t))+\lambda u(t), \quad \text { if } t \in I=[0, T] ; \\
u(0)=u(T) .
\end{gathered}
$$

Using variation of parameters formula, we can easily deduce that problem (1) is equivalent to the integral equation

$$
u(t)=\int_{0}^{T} G(t, s)[f(s, u(s))+\lambda u(s)] d s
$$

where

$$
G(t, s)= \begin{cases}\frac{e^{\lambda(T+s-t)}}{e^{\lambda T}-1}, & 0 \leq s<t \leq T \\ \frac{e^{\lambda(s-t)}}{e^{\lambda T}-1}, & 0 \leq t<s \leq T\end{cases}
$$

Define $F: C(I) \rightarrow C(I)$ by

$$
(F u)(t)=\int_{0}^{T} G(t, s)[f(s, u(s))+\lambda u(s)] d s
$$

Note that if $u \in C(I)$ is a fixed point of $F$ then $u \in C^{1}(I)$ is a solution of (1).

Now, we check that hypotheses of Corollary 13. Consider the space $X=C(I)$ with the metric

$$
d(x, y)=\|x-y\|=\max _{t \in I}|x(t)-y(t)| .
$$

Then by (28) for any $u, v \in X$,

$$
\begin{aligned}
& d(F u, F v)= \max _{t \in I}|(F u)(t)-(F v)(t)| \\
& \leq \max _{t \in I} \int_{0}^{T} G(t, s) \mid f(t, u(s))+\lambda u(s) \\
& \quad-[f(t, v(s))+\lambda v(s)] \mid d s \\
& \leq \max _{t \in I} \int_{0}^{T} G(t, s) \lambda \phi(|u(s)-v(s)|) d s .
\end{aligned}
$$

As the function $\phi(x)$ is increasing, we obtain

$$
\begin{aligned}
d(F u, F v) & \leq \max _{t \in I} \int_{0}^{T} G(t, s) \lambda \phi(|u(s)-v(s)|) d s \\
& \leq \lambda \phi(d(u, v)) \cdot \max _{t \in I} \int_{0}^{T} G(t, s) d s
\end{aligned}
$$

$$
\begin{aligned}
= & \lambda \phi(d(u, v)) \cdot \max _{t \in I} \frac{1}{e^{\lambda T}-1} \\
& \left.\left.\times\left(\frac{1}{\lambda} e^{\lambda(T+s-t)}\right]_{0}^{t}+\frac{1}{\lambda} e^{\lambda(s-t)}\right]_{t}^{T}\right) \\
= & \lambda \phi(d(u, v)) \cdot \frac{1}{e^{\lambda T}-1} \cdot \frac{1}{\lambda}\left(e^{\lambda T}-1\right) \\
= & \phi(d(u, v))=\frac{\phi(d(u, v))}{d(u, v)} \cdot d(u, v) \\
= & \beta(d(u, v)) d(u, v) .
\end{aligned}
$$

From Corollary 13, we see that $F$ has a unique fixed point.

Remark 15. Clearly, we see that the condition (6) of Theorem 2 implies the condition (28) of Theorem 14. In addition, Theorem 14 need not assume the existence of a lower solution for (1). Hence this result is an improvement of Theorem 2.

Theorem 16. Consider problem (1) with $f$ continuous and suppose that there exists $\lambda>0$ such that for any $x, y \in \mathbf{R}$

$$
|f(t, x)+\lambda x|+|f(t, y)+\lambda y| \leq \lambda \phi(|x|+|y|),
$$

where $\phi \in \mathscr{A}$. Then there exists a unique solution for (1).

Proof. Problem (1) is equivalent to the integral equation

$$
u(t)=\int_{0}^{T} G(t, s)[f(s, u(s))+\lambda u(s)] d s
$$

where $G(t, s)$ is the same as (31). Define $F: C(I) \rightarrow C(I)$ by

$$
(F u)(t)=\int_{0}^{T} G(t, s)[f(s, u(s))+\lambda u(s)] d s
$$

Notice that if $u \in C(I)$ is a fixed point of $F$ then $u \in C^{1}(I)$ is a solution of (1).

Now, we check that hypotheses of Theorem 12. Consider the space $X=C(I)$ with the metric

$$
d(x, y)=\|x-y\|=\max _{t \in I}|x(t)-y(t)|
$$

We take a $w$-distance on $X=C(I)$ by $p(x, y)=$ $\max _{t \in I}(|x(t)|+|y(t)|)$; see Example 10. Using the monotene property of the function $\phi(x)$ and condition (36), for any $u, v \in X$,

$$
\begin{aligned}
& p(F u, F v)=\max _{t \in I}(|(F u)(t)|+|(F v)(t)|) \\
& \leq \max _{t \in I}\left(\int_{0}^{T} G(t, s)(|f(t, u(s))+\lambda u(s)|\right. \\
&+|f(t, v(s))+\lambda v(s)|) d s)
\end{aligned}
$$




$$
\begin{aligned}
& \leq \max _{t \in I} \int_{0}^{T} G(t, s) \lambda \phi(|u(s)|+|v(s)|) d s \\
& \leq \lambda \phi(p(u, v)) \cdot \max _{t \in I} \int_{0}^{T} G(t, s) d s \\
& =\lambda \phi(p(u, v)) \cdot \frac{1}{\lambda} \\
& =\phi(p(u, v))=\frac{\phi(p(u, v))}{p(u, v)} \cdot p(u, v) \\
& =\beta(p(u, v)) p(u, v) .
\end{aligned}
$$

From Theorem 12, we deduce that $F$ has a unique fixed point.

Remark 17. Let $0 \leq a<1$ and $p>1$. If we denote

$$
\phi(t)=\frac{1}{2^{p-1}} \sqrt[p]{\ln \left(\frac{a}{2^{p-1}} t^{p}+1\right)}
$$

then $\phi \in \mathscr{A}$ and $\phi(t) \leq \ln (t+1)$. Thus Theorem 4 is a sepical case of Theorem 16. However, from Theorem 12 we get $p(u, u)=\max _{t \in I}(2|u(t)|)=2\|u\|=0$ if $u$ is a solution of (1). This means $u(t)=0$ for all $t \in I$. In other words, the unique solution for (1) from Theorem 16 (in particular, from Theorem 4) can only be a zero function.

Theorem 18. Consider problem (1) with $f$ continuous and suppose that there exist $\lambda, \alpha>0$ and $p>1$ with

$$
\alpha \leq\left(\frac{p \lambda\left(e^{\lambda T}-1\right)^{p}}{T^{p-1}\left(e^{p \lambda T}-1\right)}\right)^{1 / p}
$$

such that for $x, y \in \mathbf{R}$

$$
|f(t, y)+\lambda y-[f(t, x)+\lambda x]| \leq \alpha \phi(|y-x|),
$$

where $\phi \in \mathscr{A}$. Then there exists a unique solution for (1).

Proof. Problem (1) is equivalent to the integral equation

$$
u(t)=\int_{0}^{T} G(t, s)[f(s, u(s))+\lambda u(s)] d s
$$

where $G(t, s)$ is the same as (31). Define $F: C(I) \rightarrow C(I)$ by

$$
(F u)(t)=\int_{0}^{T} G(t, s)[f(s, u(s))+\lambda u(s)] d s
$$

Notice that if $u \in C(I)$ is a fixed point of $F$ then $u \in C^{1}(I)$ is a solution of (1).
Now, we check that hypotheses of Corollary 13. By (43), for any $u, v \in X$,

$$
\begin{aligned}
& d(F u, F v)= \max _{t \in I}|(F u)(t)-(F v)(t)| \\
& \leq \max _{t \in I} \int_{0}^{T} G(t, s) \mid f(t, u(s))+\lambda u(s) \\
& \quad-[f(t, v(s))+\lambda v(s)] \mid d s \\
& \leq \max _{t \in I} \int_{0}^{T} G(t, s) \alpha \phi(|u(s)-v(s)|) d s \\
&=\alpha \max _{t \in I} \int_{0}^{T} G(t, s) \phi(|u(s)-v(s)|) d s .
\end{aligned}
$$

Using the Hölder inequality in the last integral, we get

$$
\begin{aligned}
& \int_{0}^{T} G(t, s) \phi(|u(s)-v(s)|) d s \\
& \quad \leq\left(\int_{0}^{T} G(t, s)^{p} d s\right)^{1 / p}\left(\int_{0}^{T} \phi(|u(s)-v(s)|)^{q} d s\right)^{1 / q},
\end{aligned}
$$

where $q>0$ with $1 / p+1 / q=1$. The first integral gives us

$$
\begin{aligned}
\int_{0}^{T} G(t, s)^{p} d s & =\int_{0}^{t} G(t, s)^{p} d s+\int_{t}^{T} G(t, s)^{p} d s \\
& =\int_{0}^{t} \frac{e^{p \lambda(T+s-t)}}{\left(e^{\lambda T}-1\right)^{p}} d s+\int_{t}^{T} \frac{e^{p \lambda(s-t)}}{\left(e^{\lambda T}-1\right)^{p}} d s \\
& =\frac{e^{p \lambda T}-1}{p \lambda\left(e^{\lambda T}-1\right)^{p}} .
\end{aligned}
$$

As the function $\phi(x)$ is increasing and $\alpha \leq\left(p \lambda\left(e^{\lambda T}-1\right)^{p} /\right.$ $\left.T^{p-1}\left(e^{p \lambda T}-1\right)\right)^{1 / p}$, from (47) we obtain

$$
\begin{aligned}
d(F u, F v) \leq & \alpha \max _{t \in I}\left[\left(\int_{0}^{T} G(t, s)^{p} d s\right)^{1 / p}\right. \\
& \left.\times\left(\int_{0}^{T} \phi(|u(s)-v(s)|)^{q} d s\right)^{1 / q}\right] \\
\leq & \alpha\left(\frac{e^{p \lambda T}-1}{p \lambda\left(e^{\lambda T}-1\right)^{p}}\right)^{1 / p}\left(\int_{0}^{T} \phi(\|u-v\|)^{q} d s\right)^{1 / q} \\
= & \alpha \cdot\left(\frac{e^{p \lambda T}-1}{p \lambda\left(e^{\lambda T}-1\right)^{p}}\right)^{1 / p} \cdot T^{1 / q} \cdot \phi(\|u-v\|) \\
\leq & \left(\frac{p \lambda\left(e^{\lambda T}-1\right)^{p}}{T^{p-1}\left(e^{p \lambda T}-1\right)}\right)^{1 / p} \cdot\left(\frac{e^{p \lambda T}-1}{p \lambda\left(e^{\lambda T}-1\right)^{p}}\right)^{1 / p} \\
& \cdot T^{1 / q} \cdot \phi(d(u, v))
\end{aligned}
$$




$$
\begin{aligned}
& =\phi(d(u, v))=\frac{\phi(d(u, v))}{d(u, v)} \cdot d(u, v) \\
& =\beta(d(u, v)) d(u, v) .
\end{aligned}
$$

From Corollary 13, we deduce that $F$ has a unique fixed point.

From Theorem 18, we obtain the following result when we take $p=2$.

Corollary 19. Consider problem (1) with $f$ continuous and suppose that there exist $\lambda, \alpha>0$ with

$$
\alpha \leq\left(\frac{2 \lambda\left(e^{\lambda T}-1\right)}{T\left(e^{\lambda T}+1\right)}\right)^{1 / 2}
$$

such that for $x, y \in \mathbf{R}$

$$
|f(t, y)+\lambda y-[f(t, x)+\lambda x]| \leq \alpha \phi(|y-x|),
$$

where $\phi \in \mathscr{A}$. Then there exists a unique solution for (1).

Remark 20. From [6, Remark 3.3], we see that if $\phi \in \mathscr{A}$, then $\varphi(x)=\sqrt{x \phi(x)} \in \mathscr{A}$. The condition (8) of Theorem 3 implies the condition (51) of Corollary 19. In addition, Corollary 19 need not assume the existence of a lower solution for (1). Thus Corollary 19 is an improvement of Theorem 3.

Remark 21. We easily check that $\phi(x)=\sqrt{\ln \left(x^{2}+1\right)}$ and $\phi(x)=\sqrt{x^{2} /\left(x^{2}+1\right)}$ are in $\mathscr{A}$. Therefore the condition of $[8$, Theorem 3.1], [11, Theorem 25], or [7, Theorem 3.2] implies the condition (51) of Corollary 19. In addition, Corollary 19 need not assume the existence of a lower solution for (1). Thus Corollary 19 is an improvement of [8, Theorem 3.1], [11, Theorem 25], and [7, Theorem 3.2].

\section{Conflict of Interests}

The author declares that there is no conflict of interests regarding the publication of this paper.

\section{Acknowledgments}

This work was supported in part by Scientific Studies of Higher Education Institution of Inner Mongolia (NJZZ13019) and in part by Program of Higher-level talents of Inner Mongolia University (30105-125150, 30105-135117).

\section{References}

[1] J. J. Nieto and R. Rodríguez-López, "Contractive mapping theorems in partially ordered sets and applications to ordinary differential equations," Order, vol. 22, no. 3, pp. 223-239, 2005.

[2] J. J. Nieto and R. Rodríguez-López, "Existence and uniqueness of fixed point in partially ordered sets and applications to ordinary differential equations," Acta Mathematica Sinica, vol. 23, no. 12, pp. 2205-2212, 2007.
[3] J. Harjani and K. Sadarangani, "Fixed point theorems for weakly contractive mappings in partially ordered sets," Nonlinear Analysis: Theory, Methods \& Applications, vol. 71, no. 7-8, pp. 34033410, 2009.

[4] A. Amini-Harandi and H. Emami, "A fixed point theorem for contraction type maps in partially ordered metric spaces and application to ordinary differential equations," Nonlinear Analysis: Theory, Methods \& Applications, vol. 72, no. 5, pp. 22382242, 2010.

[5] M. E. Gordji and M. Ramezani, "A generalization of Mizoguchi and Takahashi's theorem for single-valued mappings in partially ordered metric spaces," Nonlinear Analysis: Theory, Methods \& Applications, vol. 74, no. 13, pp. 4544-4549, 2011.

[6] J. Caballero, J. Harjani, and K. Sadarangani, "Contractive-like mapping principles in ordered metric spaces and application to ordinary differential equations," Fixed Point Theory and Applications, vol. 2010, Article ID 916064, 9 pages, 2010.

[7] M. Eshaghi Gordji, H. Baghani, and G. H. Kim, "A fixed point theorem for contraction type maps in partially ordered metric spaces and application to ordinary differential equations," Discrete Dynamics in Nature and Society, vol. 2012, Article ID 981517, 8 pages, 2012.

[8] F. Yan, Y. Su, and Q. Feng, "A new contraction mapping principle in partially ordered metric spaces and applications to ordinary differential equations," Fixed Point Theory and Applications, vol. 2012, article 152, 2012.

[9] S. Moradi, E. Karapınar, and H. Aydi, "Existence of solutions for a periodic boundary value problem via generalized weakly contractions," Abstract and Applied Analysis, vol. 2013, Article ID 704160, 7 pages, 2013.

[10] N. Hussain, V. Parvaneh, and J. Roshan, "Fixed point results for G- $\alpha$-contractive maps with application to boundary value problems," The Scientific World Journal, vol. 2014, Article ID 585964, 14 pages, 2014.

[11] H. H. Alsulami, S. Gülyaz, E. Karapınar, and I. Erhan, "Fixed point theorems for a class of $\alpha$-admissible contractions and applications to boundary value problem," Abstract and Applied Analysis, vol. 2014, Article ID 187031, 10 pages, 2014.

[12] O. Kada, T. Suzuki, and W. Takahashi, "Nonconvex minimization theorems and fixed point theorems in complete metric spaces," Mathematica Japonica, vol. 44, no. 2, pp. 381-391, 1996. 


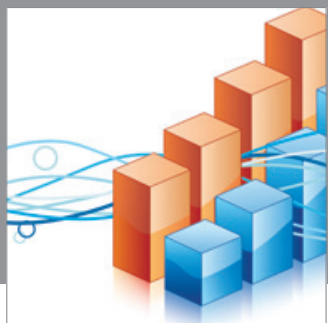

Advances in

Operations Research

mansans

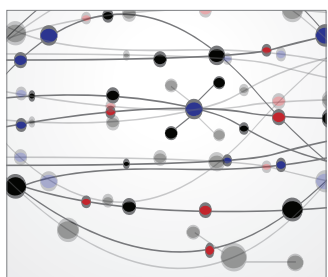

The Scientific World Journal
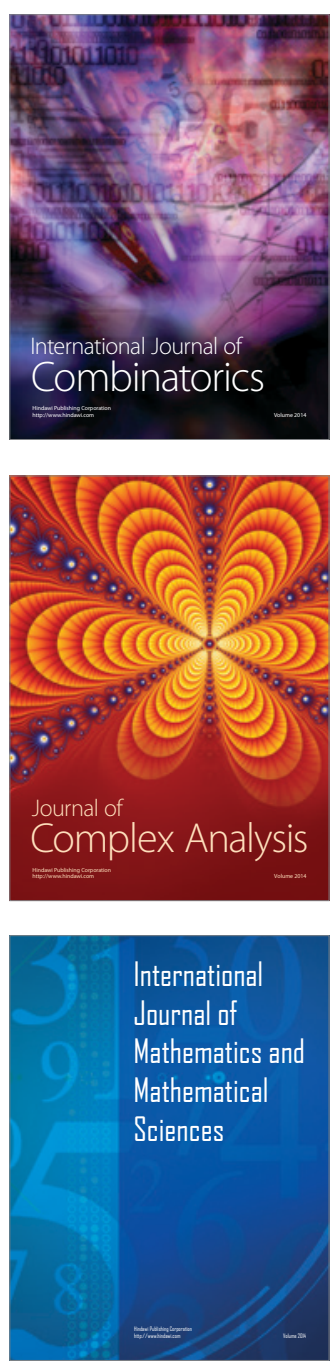
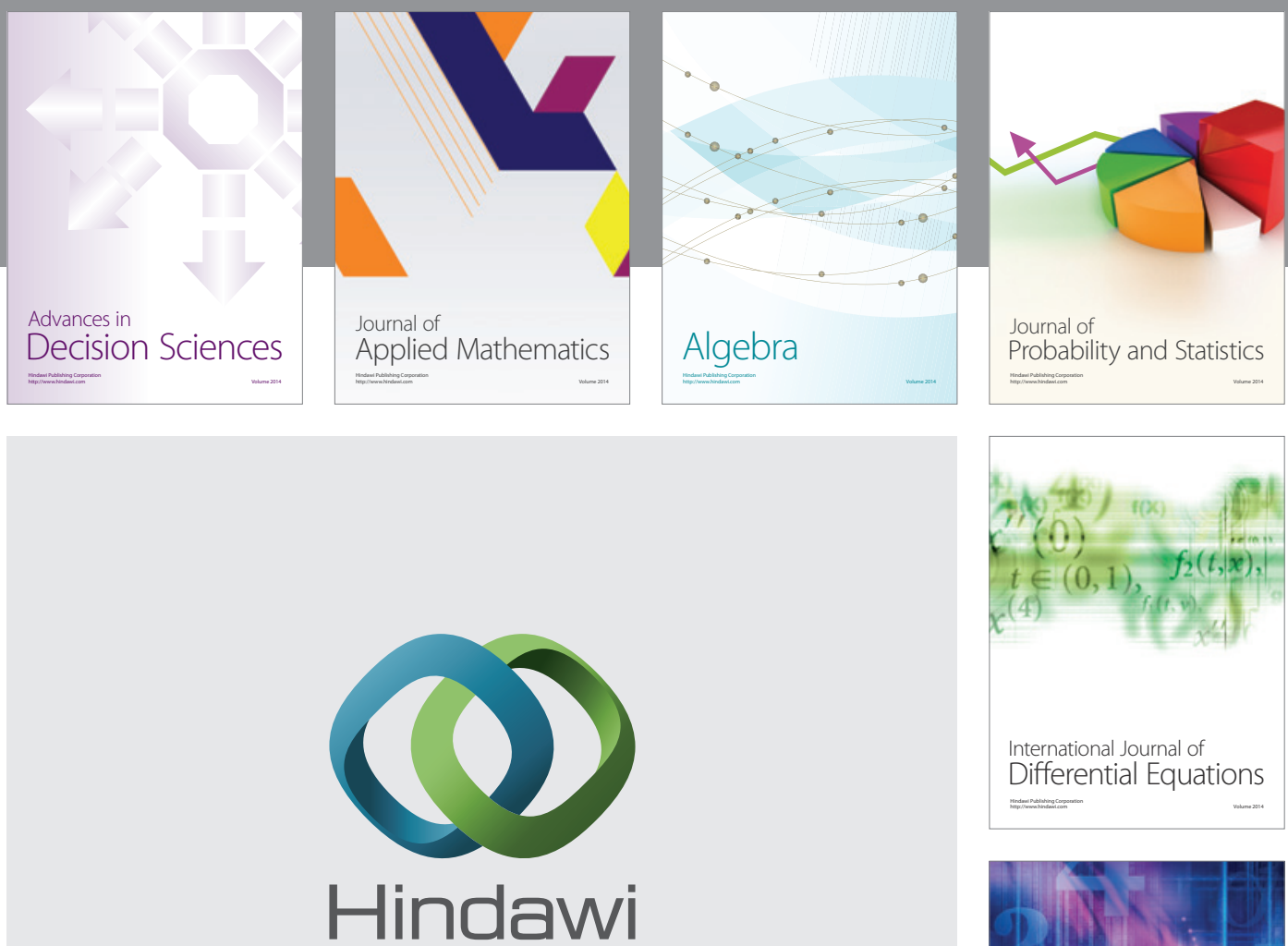

Submit your manuscripts at http://www.hindawi.com
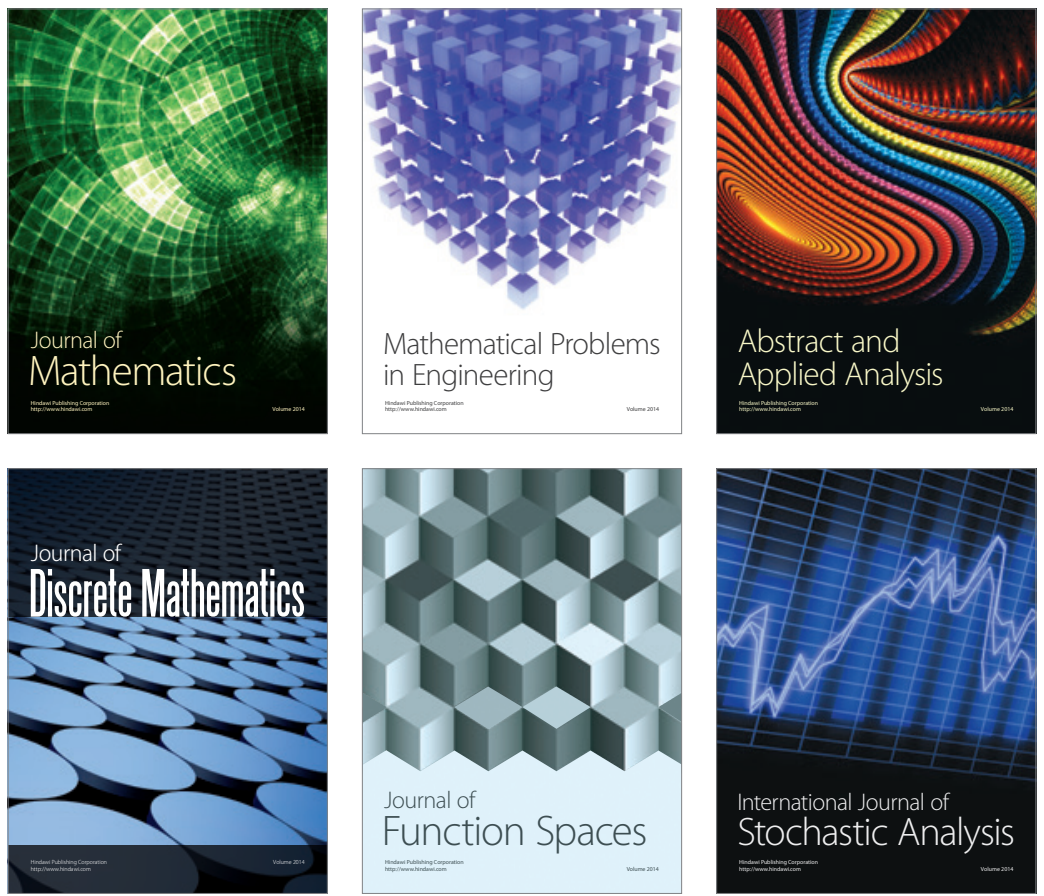

Journal of

Function Spaces

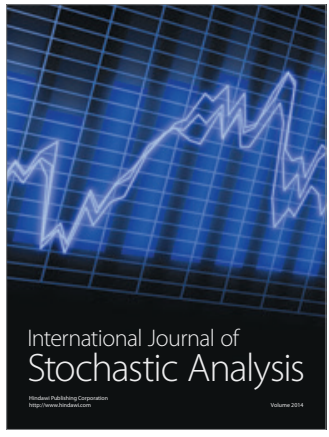

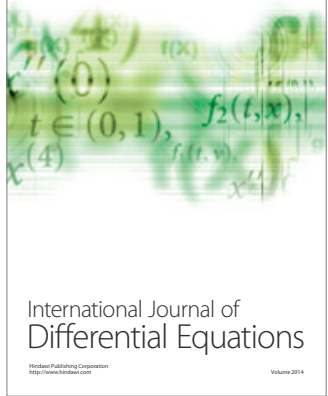
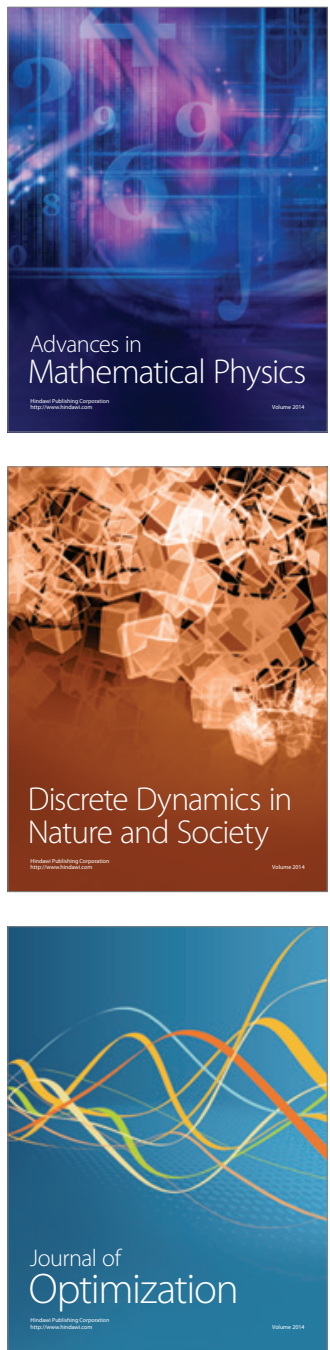